\title{
Validating the Completeness of the Real Solution Set of a System of Polynomial Equations
}

\author{
Daniel A. Brake* \\ University of Notre Dame \\ dbrake@nd.edu
}

\author{
Jonathan D. Hauenstein ${ }^{*}$ \\ University of Notre Dame \\ hauenstein@nd.edu
}

\author{
Alan C. Liddell, Jr. \\ University of Notre Dame \\ aliddel1@nd.edu
}

\begin{abstract}
Computing the real solutions to a system of polynomial equations is a challenging problem, particularly verifying that all solutions have been computed. We describe an approach that combines numerical algebraic geometry and sums of squares programming to test whether a given set is "complete" with respect to the real solution set. Specifically, we test whether the Zariski closure of that set is indeed equal to the solution set of the real radical of the ideal generated by the given polynomials. Examples with finitely and infinitely many real solutions are provided, along with an example having polynomial inequalities.
\end{abstract}

\section{INTRODUCTION}

Local solving methods, such as using Newton's method, local optimization approaches, and critical point methods as highlighted in Section 5 allow one to compute real solutions to system of equations. The typical drawback of using such local methods is the inability to verify that all real solutions have been computed. This article uses sums of squares programming to validate that a complete real solution set has been computed, that is, the Zariski closure of the given set is equal to the Zariski closure of the set of all real solutions. The method follows in the footsteps of work combining numerical and symbolic methods, particularly 4, 18.

A typical situation where one may need to test the completeness of a real solution set is computing critical points. For example, $\S 8.6$ considers computing the critical points of a potential energy landscape. In such situations, local numerical methods, e.g., 20,51, exist for locating real critical points. Our approach provides a global stopping criterion for validating that all real solutions have been identified.

A related situation is the computation of the real critical points of a projection of a solution set used in the numerical decomposition of real curves and surfaces $3,11,14,47$. The

\footnotetext{
*Research partially supported by NSF grant ACI-1460032, Army Young Investigator Program (YIP), and Sloan Research Fellowship.
}

Permission to make digital or hard copies of all or part of this work for personal or classroom use is granted without fee provided that copies are not made or distributed for profit or commercial advantage and that copies bear this notice and the full citation on the first page. Copyrights for components of this work owned by others than ACM must be honored. Abstracting with credit is permitted. To copy otherwise, or republish, to post on servers or to redistribute to lists, requires prior specific permission and/or a fee. Request permissions from permissions@ acm.org.

ISSAC '16, July 19-22, 2016, Waterloo, ON, Canada

(c) 2016 ACM. ISBN 978-1-4503-4380-0/16/07 . \$ $\$ 15.00$

DOI: http://dx.doi.org/10.1145/2930889.2930910 failure to correctly compute the set of real solutions leads to a failure in the decomposition of the real component. Hence, correct and complete computation of sets of real solutions is paramount to correctly computing the decomposition.

One approach for certifying the existence of real solutions is based on the local analysis of Newton's method using Smale's $\alpha$-theory 60 developed in 33 . Building on $\alpha$-theory, there are methods for certifying smooth continuous paths for Newton homotopies 29, 30 and general homotopies 10. For example, if a smooth path is defined by a real system of equations which has a real starting point, then the endpoint of the path must also be real.

From an algebraic viewpoint, the radical of an ideal generated by a given collection of polynomials consists of all polynomials that vanish on the solution set of the given polynomials. There are several algorithms for computing the radical of a zero-dimensional ideal - some numerical, e.g., 35, 42, 43 and some symbolic, e.g., 9, 22, When there are infinitely many solutions, one can reduce to the zero-dimensional case, for example, via $22,39$.

The real radical of an ideal generated by a given collection of polynomials with real coefficients consists of all polynomials that vanish on the real solution set of the given polynomials. There have been several proposed methods for computing the real radical of an ideal. Some are symbolic, e.g., 8 based on the primary decomposition (see also 54 64, 66, 67]). Others are numerical, based on moment matrices when the number of real solutions is finite, e.g., 40, 41 42, 43, 44. A promising approach for computing the real radical when there are infinitely many real solutions was developed in 49 providing a stopping criterion for verifying that a Pommaret basis has been computed. Other methods for computing real solutions include computing a point on each semi-algebraically connected component of the real solution set, e.g., 1, 2, 27, 57,

As discussed in 49, one key issue related to computing the real radical using semidefinite programming with moment matrices is knowing when the generated polynomials form a basis for the real radical. In our approach, we first compute a set $S$ which is a subset of the Zariski closure of the real solution set. Then, we compute polynomials that vanish on $S$. Finally, for each of the computed polynomials, we use sums of squares programming to verify that it is indeed in the real radical. Since the polynomials can be validated independently, one could easily parallelize this part of the computation. Since $S$ is contained in the Zariski closure of the real solution set, every polynomial contained in the real radical vanishes on $S$. Conversely, if every polynomial 
that vanishes on $S$ is contained in the real radical, we know that a generating set for the real radical has been computed. Hence, $S$ is complete since the Zariski closure of $S$ is equal to the Zariski closure of the real solution set of the original system of equations, i.e., the solution set of the real radical.

One of the pitfalls of using purely symbolic methods to compute real radicals is the typical computation of field extensions. As an illustrative example, consider the polynomial $f(x)=x^{3}-2$ having rational coefficients, i.e., $f \in \mathbb{Q}[x]$. Since $f=0$ has one real solution, namely $x=\sqrt[3]{2}$, the real radical of the ideal generated by $f$ is $\langle x-\sqrt[3]{2}\rangle$ which is generated by a polynomial not in $\mathbb{Q}[x]$.

By working over $\mathbb{R}$, one avoids the use of field extensions for computing the generators of the real radical. Thus, the theoretical results for our approach assume an exact computational model over $\mathbb{R}$ as in 12 . The drawback is that the computations in practice are performed using floating-point arithmetic. In the examples, we approximate the computational model by utilizing adaptive precision floating-point computations where each point in the subset $S$ of the solution set is described via a numerical approximation and an algorithm that can be used to approximate it efficiently to arbitrary precision as in 28. For example, $\alpha$-theory 60 . verifies that a numerical approximation is in the quadratic convergence basin of Newton's method and thus Newton's method can be used to efficiently refine the numerical approximation. One could then use exactness recovery methods, e.g., 5], with the refinability of numerical approximations to determine exact results.

The remainder of the article is as follows. Section 2 focuses on radicals, irreducible decomposition, and Zariski closures. Real radicals, sums of squares, and semidefinite programming are discussed in Section 3. In Section 4 we present the criterion for showing that a set $S$ is complete with respect to the real radical. This approach depends on the ability to compute such an $S$, which is highlighted in Section 5, and the ideal of $S$, which is discussed in Section 6 Section 7 considers the real solution set for collection of equations and inequalities. Several examples are presented in Section 8 with a discussion of the limitations of our approach in Section 9 . We conclude in Section 10 .

\section{Acknowledgments}

The authors thank Mohab Safey El Din, Charles Wampler, and Lihong Zhi for discussions related to real solution sets, as well as the reviewers for their helpful comments.

\section{ZARISKI CLOSURE AND RADICALS}

Let $f_{1}, \ldots, f_{k} \in \mathbb{C}\left[x_{1}, \ldots, x_{n}\right]$ and consider the ideal generated by these polynomials, namely $I=\left\langle f_{1}, \ldots, f_{k}\right\rangle$. The polynomials $f=\left\{f_{1}, \ldots, f_{k}\right\}$ and the corresponding ideal $I=\langle f\rangle$ define the same solution set in $\mathbb{C}^{n}$, namely

$$
\mathcal{V}_{\mathbb{C}}(f)=\mathcal{V}_{\mathbb{C}}(I)=\left\{x \in \mathbb{C}^{n} \mid f_{i}(x)=0 \text { for } i=1, \ldots, k\right\}
$$

A set $A \subset \mathbb{C}^{n}$ is called an algebraic set if there is a collection of polynomials $g \subset \mathbb{C}\left[x_{1}, \ldots, x_{n}\right]$ such that $A=\mathcal{V}_{\mathbb{C}}(g)$. The algebraic set $A$ is irreducible if there does not exist algebraic sets $A_{1}, A_{2} \subsetneq A$ with $A=A_{1} \cup A_{2}$. Given an algebraic set $A$, there exists a unique collection (up to relabeling) of irreducible algebraic sets $X_{1}, \ldots, X_{\ell}$ such that

$$
A=\bigcup_{i=1}^{\ell} X_{i} \text { and } X_{j} \not \subset \bigcup_{i \neq j} X_{i}
$$

Each $X_{i}$ is called an irreducible component of $A$.

In numerical algebraic geometry, an irreducible algebraic set is represented by a witness set, see, e.g., 63, Chap. 13]. A numerical irreducible decomposition for an algebraic set $A$ is a collection of witness sets for the irreducible components of $A$. Such a decomposition can be computed using various algorithms, e.g., 6, 32, 61, 62.

For any subset $T \subset \mathbb{C}^{n}$, the ideal defined by $T$ is

$$
I(T)=\left\{f \in \mathbb{C}\left[x_{1}, \ldots, x_{n}\right] \mid f(t)=0 \text { for all } t \in T\right\} .
$$

The Zariski closure of $T$ is the algebraic set $\bar{T}=\mathcal{V}_{\mathbb{C}}(I(T))$, which is the intersection of all algebraic sets that contain $T$.

For an ideal $I$, the radical of $I$ is

$$
\sqrt{I}=\left\{p \in \mathbb{C}\left[x_{1}, \ldots, x_{n}\right] \mid p^{\alpha} \in I \text { for some } \alpha \in \mathbb{Z}_{>0}\right\}
$$

with $\sqrt{I}=I\left(\mathcal{V}_{\mathbb{C}}(I)\right)$ following Hilbert's Nullstellensatz.

\section{REAL RADICAL \& SUMS OF SQUARES}

Many of the topics from $\S 2$ have analogous statements over $\mathbb{R}$. Let $f_{1}, \ldots, f_{k} \in \mathbb{R}\left[x_{1}, \ldots, x_{n}\right]$ with $f=\left\{f_{1}, \ldots, f_{k}\right\}$ and $I=\langle f\rangle$. The set of solutions in $\mathbb{R}^{n}$ is

$\mathcal{V}_{\mathbb{R}}(f)=\mathcal{V}_{\mathbb{R}}(I)=\left\{x \in \mathbb{R}^{n} \mid f_{i}(x)=0\right.$ for $\left.i=1, \ldots, k\right\}=\mathcal{V}_{\mathbb{C}}(I) \cap \mathbb{R}^{n}$.

The real radical of $I$ is

$$
\sqrt[\mathbb{R}]{I}=\left\{p \in \mathbb{R}[x] \mid \begin{array}{l}
p^{2 \alpha}+\sum_{j=1}^{\ell} g_{j}^{2} \in I \\
\text { for some } \alpha \in \mathbb{Z}_{>0}, g_{j} \in \mathbb{R}[x]
\end{array}\right\},
$$

with $\sqrt[\mathbb{R}]{I}=I\left(\mathcal{V}_{\mathbb{R}}(I)\right)$ following the real Nullstellensatz, e.g., see 13. Chap. 4].

ExAmple 1. For $f(x)=x^{3}-2$ and $I=\langle f\rangle$, we have:

- $\mathcal{V}_{\mathbb{C}}(I)=\left\{\sqrt[3]{2}, \omega \sqrt[3]{2}, \omega^{2} \sqrt[3]{2}\right\}$ and $\mathcal{V}_{\mathbb{R}}(I)=\{\sqrt[3]{2}\}$

- $\sqrt{I}=I$, and

- $\sqrt[\mathbb{R}]{I}=\langle x-\sqrt[3]{2}\rangle$

where $\omega$ is the primitive cube root of unity. In particular,

$$
(x-\sqrt[3]{2})^{4}+\left(\sqrt{3} x^{2}-\sqrt{3} \sqrt[3]{4}\right)^{2}=4\left(x^{3}-2\right)(x-\sqrt[3]{2}) \in I .
$$

The algebraic description of the real radical $\sqrt[\mathbb{R}]{I}$ presented in (1) shows that this definition depends on sums of squares. A polynomial $s \in \mathbb{R}\left[x_{1}, \ldots, x_{k}\right]$ is called a sum of squares if $s=\sum_{j=1}^{\ell} g_{j}^{2}$ for some $g_{1}, \ldots, g_{\ell} \in \mathbb{R}\left[x_{1}, \ldots, x_{k}\right]$. Clearly, every polynomial that is a sum of squares has even degree.

The polynomials of even degree that are sums of squares are characterized by positive semidefinite matrices. A symmetric matrix $M \in \mathbb{R}^{m \times m}$ is positive semidefinite if, for all $y \in \mathbb{R}^{m}, y^{T} M y \geq 0$. This condition is equivalent to all eigenvalues of $M$ being nonnegative. We will write $M \succeq 0$ if $M$ is positive semidefinite.

Let $s \in \mathbb{R}\left[x_{1}, \ldots, x_{k}\right]$ be a polynomial of degree $2 d$ and $X_{d}$ be the vector of all monomials in $x_{1}, \ldots, x_{n}$ of degree at most $d$. Hence, there exists a symmetric matrix $C$ such that

$$
s(x)=X_{d}^{T} \cdot C \cdot X_{d} .
$$

The polynomial $s$ is a sum of squares if and only if there is a positive semidefinite matrix $C$ such that (2) holds, e.g., 16.

EXAMPLE 2. As shown in Ex. 1, the quartic polynomial $s(x)=4\left(x^{3}-2\right)(x-\sqrt[3]{2})$ is a sum of squares. Let

$$
X_{2}=\left[\begin{array}{c}
1 \\
x \\
x^{2}
\end{array}\right] \text { and } C=\left[\begin{array}{ccc}
8 \sqrt[3]{2} & -4 & -2 \sqrt[3]{4} \\
-4 & 4 \sqrt[3]{4} & -2 \sqrt[3]{2} \\
-2 \sqrt[3]{4} & -2 \sqrt[3]{2} & 4
\end{array}\right]
$$

It is easy to verify that $C \succeq 0$ and $s(x)=X_{2}^{T} \cdot C \cdot X_{2}$. 
For a given polynomial $s$ of degree $2 d$, the set of symmetric matrices $C$ such that 2 holds is a linear space. Hence, testing that a polynomial is a sum of squares can be accomplished by solving a semidefinite feasibility problem.

EXAmple 3. Continuing with $s(x)=4\left(x^{3}-2\right)(x-\sqrt[3]{2})$ from Ex. 2, consider the linear space

$$
\mathcal{L}=\left\{\left[\begin{array}{lll}
s_{00} & s_{01} & s_{02} \\
s_{01} & s_{11} & s_{12} \\
s_{02} & s_{12} & s_{22}
\end{array}\right] \mid \begin{array}{rl}
s_{00} & =8 \sqrt[3]{2} \\
2 s_{01} & =-8 \\
2 s_{02}+s_{11} & =0 \\
2 s_{12} & =-4 \sqrt[3]{2} \\
s_{22} & =4
\end{array}\right\}
$$

Since $s(x)=X_{2}^{T} \cdot C \cdot X_{2}$ if and only if $C \in \mathcal{L}$, it follows that $s$ is a sum of squares if and only if there exists $C \in \mathcal{L}$ such that $C \succeq 0$, which is a semidefinite feasibility problem.

To facilitate the task of converting between sums of squares problems and semidefinite programming problems in the examples, we utilize the software package SOSTOOLS 55.

Given a polynomial $p \in \mathbb{R}\left[x_{1}, \ldots, x_{n}\right]$, we can decide if $p \in \sqrt[\mathbb{R}]{I}$ using $(1)$. That is, $p \in \sqrt[\mathbb{R}]{I}$ if and only if there exists $\alpha \in \mathbb{Z}_{>0}$ and $h_{1}, \ldots, h_{k}, g_{1}, \ldots, g_{\ell} \in \mathbb{R}\left[x_{1}, \ldots, x_{n}\right]$ such that

$$
p^{2 \alpha}+\sum_{j=1}^{\ell} g_{j}^{2}=\sum_{i=1}^{k} h_{i} f_{i}
$$

which is equivalent to requiring that

$$
-p^{2 \alpha}+\sum_{i=1}^{k} h_{i} f_{i} \text { is a sum of squares. }
$$

Thus, given a polynomial $p \in \mathbb{R}\left[x_{1}, \ldots, x_{n}\right]$, one can test if $p \in \sqrt[\mathbb{R}]{I}$ by solving a semidefinite feasibility problem. We use this observation in our method, which is described next.

\section{VALIDATION}

Given an ideal $I \subset \mathbb{R}\left[x_{1}, \ldots, x_{n}\right], S \subset \overline{\mathcal{V}_{\mathbb{R}}(I)}=\mathcal{V}_{\mathbb{C}}(\sqrt[\mathbb{R}]{I})$, and $I(S)=\left\langle g_{1}, \ldots, g_{\ell}\right\rangle$, we describe an approach for validating that $S$ is complete, i.e., $S=\overline{\mathcal{V}_{\mathbb{R}}(I)}$ which occurs if and only if $\sqrt[\mathbb{R}]{I}=\left\langle g_{1}, \ldots, g_{\ell}\right\rangle=I(S)$.

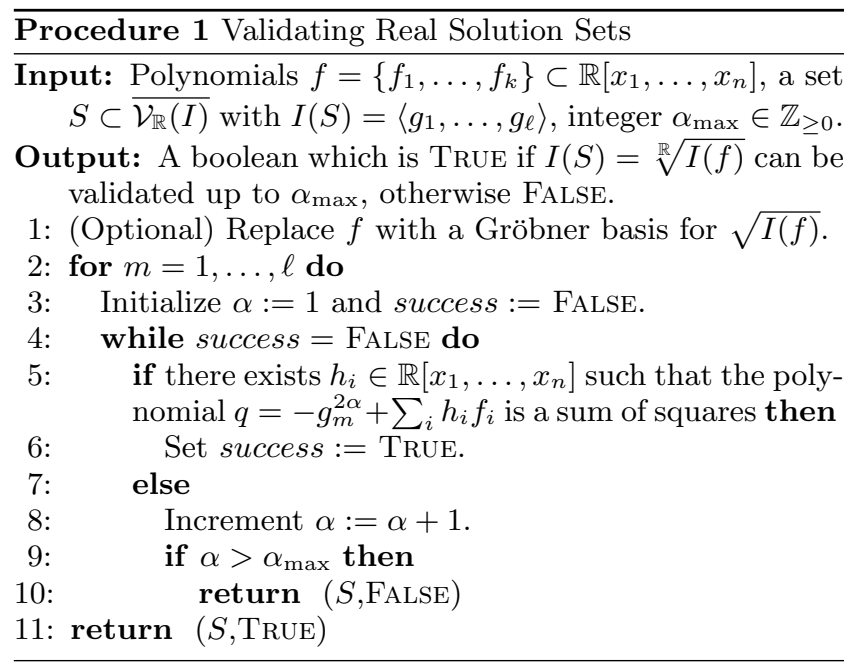

TheOREm 4. Procedure 1 is a correct algorithm.
Proof. Let $I=\left\langle f_{1}, \ldots, f_{k}\right\rangle$. Since $\mathcal{V}_{\mathbb{R}}(I)=\mathcal{V}_{\mathbb{R}}(\sqrt{I})$, replacing $f$ by a Gröbner basis for $\sqrt{I}$ does not impact the set of real solutions.

For a given $p \in \mathbb{R}\left[x_{1}, \ldots, x_{n}\right]$, we know $p \in \sqrt[\mathbb{R}]{I}$ if and only if there exists $\alpha \in \mathbb{Z}_{>0}$ and $h_{1}, \ldots, h_{k} \in \mathbb{R}\left[x_{1}, \ldots, x_{n}\right]$ such that (3) holds. In particular, since $S \subset \overline{\mathcal{V}_{\mathbb{R}}(I)}$, we know $\sqrt[\mathbb{R}]{I} \subset I(S)=\left\langle g_{1}, \ldots, g_{\ell}\right\rangle$ so that $\sqrt[\mathbb{R}]{I}=I(S)$ if and only if (3) holds for each $p=g_{i}$. If all of the corresponding $\alpha$ 's for each $g_{i}$ are at most $\alpha_{\max }$, then Procedure 1 will correctly determine $I(S)=\sqrt[\mathbb{R}]{I}$.

If $p \notin \sqrt[\mathbb{R}]{I}$, then, for every $\alpha \in \mathbb{Z}_{>0}, \sqrt{3}$ does not hold. In Procedure 1, we use the upper bound $\alpha_{\max }$ so that Procedure 1 always terminates.

If one could compute an a priori upper bound on the largest possible value for $\alpha$, then we could replace $\alpha_{\max }$ with this bound. However, without such a bound, we simply keep searching for new points to add to $S$, which is described next. If $p \notin \sqrt[\mathbb{R}]{I}$, then there must exist a point $x \in \mathcal{V}_{\mathbb{R}}(I)$ such that $p(x) \neq 0$. In fact, there is an irreducible component $X \subset \overline{\mathcal{V}_{\mathbb{R}}(I)}=\mathcal{V}_{\mathbb{C}}(\sqrt[\mathbb{R}]{I})$ such that $p(x) \neq 0$ for every $x$ in a dense open subset of $X$.

We have implemented this procedure using floating-point arithmetic computations for several example systems ${ }^{1}$ while a full general-purpose implementation is left for future work.

\section{GENERATING A CANDIDATE SET}

Input for Procedure 1 is a set $S$ and generators for $I(S)$. Hence, in this section we provide techniques for generating $S$ with the next focusing on computing $I(S)$.

\subsection{Approaches for locating real solutions}

A classical approach for attempting to find a real solution is to use Newton's method or related variants, see, e.g., 38. For a polynomial system with real coefficients, if the initial point is real, then every solution obtained from Newton's method is also real. Of course, there are many challenges associated with finding real solutions using Newton's method, particularly when $\mathcal{V}_{\mathbb{C}}(f)$ is not a complete intersection or the real solutions are singular with respect to $f$. That is, problems can occur with Newton's method, e.g., divergence, if the dimension of the solution set is less than dimension of the null space of the Jacobian at the solution 23, 24]. Nonetheless, heuristic techniques such as damping methods, reusing Jacobians for several iterations, or using chord or secant methods can be utilized 38].

Another approach for computing real solutions is to utilize numerical optimization techniques. Standard iterative techniques include those based on nonlinear least squares approaches such as the Levenberg-Marquardt algorithm and alternating least squares 37]. Other standard methods in optimization include the worker bees method, genetic algorithms, and the Nelder-Mead method, see, e.g., 17.

Critical point methods combine optimization and polynomial system solving techniques. For example, Seidenberg [58] considered the critical points of the distance function between the set of real solutions and a given real point $y^{*}$ that was not a solution. The set of all such critical points contains a point on every connected component of the real solution set 1, 56, 58. By utilizing homotopy continuation, one can compute a finite subset of critical points containing

\footnotetext{
${ }^{1}$ Available at www.nd.edu/ aliddel1/validate-reals
} 
a point on every connected component 27]. Moreover, one can then sample more real points by moving $y^{*}$.

Rather than compute all critical points, one can attempt to compute the closest critical point to the given $y^{*}$. This can be accomplished using a classical optimization approach such as the gradient descent method or a homotopy-based approach called gradient-descent homotopy 25. By testing at many values of $y^{*}$, one aims to quickly generate many real solutions, e.g., as shown in 25, Fig. 3].

Other so-called "local" solving methods exist for finding real solutions, which have been used in various disciplines. Some examples include techniques in theoretical chemistry, e.g., 20,51, 52 and solving power-flow equations in electrical engineering, e.g., 45, 48.

\subsection{Real solutions and isosingular sets}

After a real solution has been located, one can now try to extract additional information about the geometry of the solution set near this point. One approach is to compute a local irreducible decomposition using local witness sets 15 to see if local structure provides insight into the components of the real solution set passing through the computed real point. Another approach is to utilize isosingular sets [34], which may also help in improving the numerical stability of interpolation, described in the next section.

Let $f_{1}, \ldots, f_{k}$ be polynomials and $z \in \mathcal{V}_{\mathbb{C}}(f)$. Let $J f(z)$ be the Jacobian matrix of $f$ evaluated at $z$. For an integer $\ell$, let $\operatorname{det}_{\ell} J f(z)$ be the collection of all $(\ell+1) \times(\ell+1)$ minors of $J f(z)$. Thus, $\operatorname{det}_{\ell} J f(z)=0$ if and only if rank $J f(z) \leq \ell$. For a polynomial system $g$, let $\operatorname{dnull}(g, z)=\operatorname{dim}$ null $J g(z)$. The deflation sequence of $z$ with respect to $f$ is defined by

$$
d_{i}(f, z)=\operatorname{dnull}\left(\mathcal{D}^{i}(f, z), z\right) \text { for } i \in \mathbb{Z}_{\geq 0}
$$

where $\mathcal{D}^{0}(f, z)=f$ and

$$
\mathcal{D}^{i}(f, z)=\left[\begin{array}{c}
\mathcal{D}^{i-1}(f, z) \\
\operatorname{det}_{d_{i-1}(f, z)} J \mathcal{D}^{i-1}(f, z)
\end{array}\right] .
$$

The deflation sequence is a nonincreasing sequence of nonnegative integers and thus has a limit, say $d_{\infty}(f, z) \geq 0$, called the isosingular local dimension of $z$ with respect to $f$.

Due to potential issues with using minors, e.g., the total number of them and the potential for each to have very high degree, there are alternative approaches. For example, a so-called strong deflation approach based on the null space method of 19,46 , was presented in [34, and a variant involving polynomial many new functions proposed in [31.

If $X(f, z)$ is the Zariski closure of all points in $\mathcal{V}_{\mathbb{C}}(f)$ which have the same deflation sequence with respect to $f$ as $z$, then 34. Lemma 5.14] yields that there is a unique irreducible component of $X(f, z)$ which contains $z$, denoted $\operatorname{Iso}_{f}(z)$, called the isosingular set of $z$ with respect to $f$. In particular, $d_{\infty}(f, z)=\operatorname{dim} \operatorname{Iso}_{f}(z)$.

Suppose that $z \in \mathcal{V}_{\mathbb{R}}(f) \subset \mathbb{R}^{n}$. Since $z$ is a smooth point on the irreducible set $\operatorname{Iso}_{f}(z)$, we have $\operatorname{Iso}_{f}(z) \cap \mathbb{R}^{n} \subset \mathcal{V}_{\mathbb{R}}(f)$ and $\operatorname{Iso}_{f}(z)=\overline{\operatorname{Iso}_{f}(z) \cap \mathbb{R}^{n}} \subset \overline{\mathcal{V}_{\mathbb{R}}(f)}$. That is, if $I=\langle f\rangle$,

$$
\operatorname{Iso}_{f}(z) \subset \mathcal{V}_{\mathbb{C}}(\sqrt[\mathbb{R}]{I}) \text { and } \sqrt[\mathbb{R}]{I} \subset I\left(\operatorname{Iso}_{f}(z)\right) .
$$

The isosingular local dimension is a lower bound on the local real dimension at $z$, which is $\operatorname{sharp}$ if $z$ is a smooth point on a unique irreducible component of $\mathcal{V}_{\mathbb{C}}(\sqrt[\mathbb{R}]{I})$. Moreover, if $d_{\infty}(f, z)>0$, we can use standard sampling techniques in numerical algebraic geometry, see, e.g., [7, § 8.3], applied to
$\operatorname{Iso}_{f}(z)$ to produce an arbitrary number of additional points for which polynomials in $\sqrt[\mathbb{R}]{I}$ must vanish.

Additionally, by using isosingular sets and numerical algebraic geometry, we can utilize standard membership tests, see, e.g., [7, $§ 8.4]$, to determine if a newly found point $x \in \mathcal{V}_{\mathbb{R}}(I)$ is already contained in the set $S$.

\section{INTERPOLATION}

From the set $S \subset \overline{\mathcal{V}_{\mathbb{R}}(I)}$ constructed in $\S 5$, the next task is to compute a collection of polynomials which vanish on $S$ with Procedure 1 testing whether $I(S)$ is equal to $\sqrt[\mathbb{R}]{I}$.

Suppose that $T \subset \mathbb{C}^{n}$ is a finite set such that $I(T)$ is generated by real polynomials and $d \geq 1$. Let $\mathcal{B}$ form a basis for the finite-dimensional vector space of all polynomials in $n$ variables with real coefficients of degree at most $d$, namely $\mathbb{R}\left[x_{1}, \ldots, x_{n}\right]_{\leq d}$. The linear space of polynomials of degree at most $d$ in $I(T)$, denoted $I(T)_{\leq d}$, is (isomorphic to) the null space of matrix $M$ where $M_{i j}=\beta_{j}\left(t_{i}\right)$, i.e., the evaluation of the $j^{\text {th }}$ basis element $\beta_{j} \in \mathcal{B}$ at the $i^{\text {th }}$ point $t_{i} \in T$. If $S$ is a finite set, then we simply take $T=S$. Otherwise, one can take $T$ to be a finite set consisting of sufficiently many points on each irreducible component described by $S$. The number of sample points needed on each component can be a priori bounded based on the dimension of $\mathbb{R}\left[x_{1}, \ldots, x_{n}\right]_{\leq d}$. One can also algorithmically bound the number of sample points needed per component simply by continuing to add sample points from each component to $T$ until the rank of the associated matrix $M$ stabilizes.

As shown in 26, one can rescale each row independently to improve the conditioning of interpolation. Moreover, for positive-dimensional components, sampling points that are spread out over the component using numerical algebraic geometry as in $\S 5.2$ also helps to improve conditioning.

EXAMPLE 5. The solution set of the polynomial system

$$
f=\left\{x^{2}+y^{2}+z^{2}-1, x^{2}+y^{2}+z-1, x\right\}
$$

consists of the three points

$$
\mathcal{V}_{\mathbb{C}}(f)=\mathcal{V}_{\mathbb{R}}(f)=\{(0,1,0),(0,-1,0),(0,0,1)\}
$$

where the point $(0,0,1)$ has multiplicity two with respect to $f$.

To illustrate, for $d=2$, we choose the monomial basis

$$
\mathcal{B}=\left\{1, x, y, z, x^{2}, x y, x z, y^{2}, y z, z^{2}\right\}
$$

for $\mathbb{R}[x, y, z]_{\leq 2}$ with $S=T=\mathcal{V}_{\mathbb{R}}(f)$ where $M$ is

\begin{tabular}{r|rrrrrrrrrr} 
& 1 & $x$ & $y$ & $z$ & $x^{2}$ & $x y$ & $x z$ & $y^{2}$ & $y z$ & $z^{2}$ \\
\hline$(0,1,0)$ & 1 & 0 & 1 & 0 & 0 & 0 & 0 & 1 & 0 & 0 \\
$(0,-1,0)$ & 1 & 0 & -1 & 0 & 0 & 0 & 0 & 1 & 0 & 0 \\
$(0,0,1)$ & 1 & 0 & 0 & 1 & 0 & 0 & 0 & 0 & 0 & 1
\end{tabular}.

$A$ basis for null $M$ is given by the columns of the matrix

$\left[\begin{array}{rrrrrrr}0 & 0 & 0 & 0 & -1 & 0 & 0 \\ 1 & 0 & 0 & 0 & 0 & 0 & 0 \\ 0 & 0 & 0 & 0 & 0 & 0 & 0 \\ 0 & 0 & 0 & 0 & 1 & 0 & -1 \\ 0 & 1 & 0 & 0 & 0 & 0 & 0 \\ 0 & 0 & 1 & 0 & 0 & 0 & 0 \\ 0 & 0 & 0 & 1 & 0 & 0 & 0 \\ 0 & 0 & 0 & 0 & 1 & 0 & 0 \\ 0 & 0 & 0 & 0 & 0 & 1 & 0 \\ 0 & 0 & 0 & 0 & 0 & 0 & 1\end{array}\right]$

corresponding to the polynomials

$$
x, x^{2}, x y, x z, y^{2}+z-1, y z, z^{2}-z
$$


which form a basis for the linear space $(\sqrt[\mathbb{R}]{I})_{\leq 2}$. Note that since each polynomial $f_{i}$ has degree at most 2 , each $f_{i}$ is contained in the linear span of these polynomials.

For illustrative purposes, we selected a monomial basis. In practice, the choice of basis should be made based on numerical conditioning.

For $d \gg 0$, we know $I(S)=\left\langle I(S)_{\leq d}\right\rangle$. If $S$ is a finite set, then one can determine an upper bound on $d$ such that $I(S)$ is generated by $I(S)_{\leq d}$. In particular, the function

$$
c \mapsto \operatorname{dim} \mathbb{R}\left[x_{1}, \ldots, x_{n}\right]_{\leq c}-\operatorname{dim} I(S)_{\leq c}
$$

is the Hilbert function of $I(S)$. If $r$ is the minimum such that $|S|=\operatorname{dim} \mathbb{R}\left[x_{1}, \ldots, x_{n}\right]_{\leq r}-\operatorname{dim} I(S)_{\leq r}$, i.e., the index of regularity, then one knows that $I(S)$ is either generated by $I(S)_{\leq r}$ or $I(S)_{\leq r+1}$. In fact, $I(S)_{\leq r}$ generates $I(S)$ if and only if $\left\langle I(S)_{\leq r}\right\rangle_{\leq r+1}=I(S)_{\leq r+1}$, i.e., the Hilbert function of $J=\left\langle I(S)_{\leq r}\right\rangle$ in degree $r+1$ is also equal to $|S|$.

EXAmple 6. Continuing with Ex.5, since

$$
\operatorname{dim} \mathbb{R}[x, y, z]_{\leq 2}-\operatorname{dim} I(S)_{\leq 2}=10-7=3=|S|,
$$

one can easily verify that $I(S)$ is generated by $I(S)_{\leq 2}$, i.e.,

$$
\sqrt[\mathbb{R}]{I}=\left\langle x, y^{2}+z-1, y z, z^{2}-z\right\rangle .
$$

EXAMPLE 7. The Hilbert function for the ideal $I(S)$ where $S=\{(0,0),(0,1),(1,0)\}$ is $1,3,3, \ldots$ so that $I(S)$ is either generated by $I(S)_{\leq 1}$ or $I(S)_{\leq 2}$. Since $I(S)_{\leq 1}=\{0\}$, we know that $I(S)_{\leq 2}$ must generate $I(S)$.

When $S$ is infinite, we aim to reduce our computations to standard computations performed over $\mathbb{C}$ as summarized in $\S 2$ In particular, by using isosingular sets as discussed in $\S \overline{5.2}$, we can actually assume that $S=\bar{S}$ and that we have a numerical irreducible decomposition of $S$. Hence, we simply need to compute $d$ large enough so that $S$ and $\mathcal{V}_{\mathbb{C}}\left(I(S)_{\leq d}\right)$ have the same irreducible components so that $S=\mathcal{V}_{\mathbb{C}}\left(I(S)_{\leq d}\right)$. Hence, $I(S)=\sqrt{\left\langle I(S)_{\leq d}\right\rangle}$.

\section{EQUALITIES AND INEQUALITIES}

One can naturally generalize from real radicals of systems of polynomial equations to $\mathcal{A}$-radicals of systems of polynomial equations and inequalities. In particular, let $f_{1}, \ldots, f_{k}, r_{1}, \ldots, r_{s} \in \mathbb{R}\left[x_{1}, \ldots, x_{n}\right]$ with

$$
I=\left\langle f_{1}, \ldots, f_{k}\right\rangle \text { and } \mathcal{A}=\left\{x \in \mathbb{R}^{n} \mid r_{i}(x) \geq 0 \text { for all } i=1, \ldots, s\right\} .
$$

The $\mathcal{A}$-radical of $I$ is $\sqrt[\mathcal{A}]{I}=I\left(\mathcal{V}_{\mathbb{R}}(I) \cap \mathcal{A}\right)$. Algebraically, one can characterize $\sqrt[A]{I}$ using sums of squares 50,65 :

$$
\sqrt[A]{I}=\left\{\begin{array}{l|l}
p \in \mathbb{R}[x] & \begin{array}{c}
p^{2 \alpha}+\sum_{\nu \in\{0,1\}^{s}} \sigma_{\nu} \cdot \prod_{j=1}^{s} r_{j}^{\nu_{j}} \in I \\
\text { for some } \alpha \in \mathbb{Z}_{>0}, \\
\text { sum of squares } \sigma_{\nu} \in \mathbb{R}[x]
\end{array}
\end{array}\right\} .
$$

Rather than try to locate sample points that satisfy equalities and inequalities, we will instead reduce to equations by introducing "slack" variables. That is, we consider the ideal

$$
J=\left\langle f_{1}(x), \ldots, f_{k}(x), r_{1}(x)-y_{1}^{2}, \ldots, r_{s}(x)-y_{s}^{2}\right\rangle .
$$

Since $\mathcal{V}_{\mathbb{R}}(I) \cap \mathcal{A}=\pi\left(\mathcal{V}_{\mathbb{R}}(J)\right)$ where $\pi(x, y)=x$, we know

$$
\sqrt[\mathcal{A}]{I}=\sqrt[\mathbb{R}]{J} \cap \mathbb{R}\left[x_{1}, \ldots, x_{n}\right]
$$

Thus, we compute $S \subset \overline{\mathcal{V}_{\mathbb{R}}(J)}$ but only perform interpolation on $\pi(S)$. If $\left\langle g_{1}, \ldots, g_{\ell}\right\rangle=I(\pi(S)) \subset \mathbb{R}\left[x_{1}, \ldots, x_{n}\right]$ and each $g_{i} \in \sqrt[\mathbb{R}]{J}$, then $I(\pi(S))=\sqrt[A]{I}$ by $(6)$.

\section{EXAMPLES}

As mentioned in the Introduction, the following examples were computed using floating-point arithmetic operations. By using approaches described in Section 5 each numerical approximation of a solution naturally comes with an algorithm which can be used to refine the approximation to arbitrary accuracy. The scripts needed to run these examples are available at www.nd.edu/ aliddel1/validate-reals

\subsection{An illustrative example}

To illustrate our approach, we consider the intersection of a circle and a bivariate cubic, namely

$$
f=\left\{x^{2}+y^{2}-2,2 x y^{2}-x+1\right\} .
$$

The system $f=0$ has six solutions, all of which are real:

$$
\mathcal{V}_{\mathbb{R}}(f)=\{(-1, \pm 1),(1.366, \pm 0.366),(-0.366, \pm 1.366)\} .
$$

In our first test, we simply take $S=\mathcal{V}_{\mathbb{R}}(f)$. Since the Hilbert function of $I(S)$ is $1,3,5,6,6, \ldots$, we can show that $I(S)$ is generated by $I(S)_{\leq 3}$. A basis for the linear space $I(S)_{\leq 3}$, computed as in $\S 6$, is:

$$
G=\left\{\begin{array}{l}
y^{3}+x^{2} y-2 y, x y^{2}-x / 2+1 / 2, \\
x^{3}-3 x / 2-1 / 2, x^{2}+y^{2}-2
\end{array}\right\} .
$$

Using either $f$ or a Gröbner basis for $\langle f\rangle$, e.g.,

$$
\left\{x^{2}+y^{2}-2,2 x y^{2}-x+1,2 y^{4}-5 y^{2}-x+2\right\},
$$

every $g \in G$ was found to be in $\sqrt[\mathbb{R}]{\langle f\rangle}$ showing that $S$ is indeed equal to $\mathcal{V}_{\mathbb{R}}(f)$.

\section{Incomplete solution set}

Suppose that we take $R=\mathcal{V}_{\mathbb{R}}(f) \cap\{y \geq 0\}$. Since the Hilbert function of $I(R)$ is $1,3,3, \ldots$ and $I(R)_{\leq 1}=\{0\}$, we know that $I(R)$ is generated by three quadratics, approximately

$$
G=\left\{\begin{array}{l}
y^{2}-2.049 y-0.18301 x+0.86603, \\
x y-0.18301 y-0.68301 x+1 / 2 \\
x^{2}+0.18301 x+2.049 y-2.866
\end{array}\right\} .
$$

Using $\alpha_{\max }=5$, we were unable to validate that any of the polynomials in $G$ where in $\sqrt[\mathbb{R}]{\langle f\rangle}$. In fact, we can show that this is indeed correct since each polynomial in $G$ is nonzero at each of the three points in $\mathcal{V}_{\mathbb{R}}(f) \backslash R$.

\section{Semialgebraic condition}

We now validate that $R=\mathcal{V}_{\mathbb{R}}(f) \cap\{y \geq 0\}$ is the complete solution set for the $\mathcal{A}$-radical of $\langle f\rangle$ where $\mathcal{A}=\{y \geq 0\}$. To that end, we add a slack variable $z$ and consider the system

$$
F=\left\{x^{2}+y^{2}-2,2 x y^{2}-x+1, y-z^{2}\right\} .
$$

As described in $\S 7$, we just need to show that each polynomial in $G$ from $\sqrt{80}$ is contained in $\sqrt[\mathbb{R}]{\langle F\rangle}$. Using either $F$ or a Gröbner basis for $\langle F\rangle$, namely (7) together with $y-z^{2}$, we validated that $G \subset \sqrt[\mathbb{R}]{\langle F\rangle}$ showing that $R$ is indeed equal to $\mathcal{V}_{\mathbb{R}}(f) \cap\{y \geq 0\}$, i.e., $\sqrt[\mathcal{A}]{\langle f\rangle}=I(R)$.

\subsection{Positive-dimensional components}

To illustrate the approach on a system such that the real radical ideal is positive-dimensional, consider the system

$$
f=\left\{x y z, z\left(x^{2}+y^{2}+z^{2}+y\right), y(y+z)\right\} .
$$


The set $\mathcal{V}_{\mathbb{C}}(f)$ consists of three lines, two of which are complex conjugates of each other that intersect at the origin and the other is a double line with respect to $f$, and an isolated point. In particular, $\mathcal{V}_{\mathbb{R}}(f)$ is the line $y=z=0$ and the isolated point $(0,-1 / 2,1 / 2)$. So, we take

$$
S=\{(x, 0,0) \mid x \in \mathbb{C}\} \cup\{(0,-1 / 2,1 / 2)\} \subset \overline{\mathcal{V}_{\mathbb{R}}(f)} .
$$

To simplify the real computations later, we first replace $f$ with a Gröbner basis for the radical $\sqrt{\langle f\rangle}$, namely

$$
f=\left\{2 y z-y, 2 y^{2}+y, x y, 4 x^{2} z+4 z^{3}+y\right\} .
$$

With the isolated solution, sampling 3 points on the line is enough to compute a basis for $I(S)_{\leq 2}$ which generates $I(S)$ :

$$
G=\left\{z^{2}+y / 2, y z-y / 2, y^{2}+y / 2, x z, x y, y+z\right\} .
$$

Each element in $G$ was shown to belong to $\sqrt[\mathbb{R}]{\langle f\rangle}$ with $\alpha \leq 2$.

\subsection{Katsura-5 system}

As an illustration of our approach on a problem which was solved using the semidefinite characterization of the real radical in 41], we consider the Katsura-5 system as in [41. Ex. 5.4]. The system consists of a linear, say $f_{1}$, and five quadratics, say $f_{2}, \ldots, f_{6}$, in six variables, namely

$$
f=\left\{\begin{array}{c}
x_{1}+2\left(x_{2}+x_{3}+x_{4}+x_{5}+x_{6}\right)-1 \\
x_{1}^{2}+2\left(x_{2}^{2}+x_{3}^{2}+x_{4}^{2}+x_{5}^{2}+x_{6}^{2}\right)-x_{1} \\
2\left(x_{1} x_{2}+x_{2} x_{3}+x_{3} x_{4}\right)+x_{4} x_{5}+x_{5} x_{6}-x_{2}, \\
x_{2}^{2}+2\left(x_{1} x_{3}+x_{2} x_{4}+x_{3} x_{5}+x_{4} x_{6}\right)-x_{3} \\
2\left(x_{1} x_{4}+x_{2} x_{3}+x_{2} x_{5}+x_{3} x_{6}\right)-x_{4} \\
x_{3}^{2}+2\left(x_{1} x_{4}+x_{1} x_{5}+x_{1} x_{6}\right)-x_{5}
\end{array}\right\} .
$$

The set $\mathcal{V}_{\mathbb{C}}(f)$ consists of 32 points, 12 of which lie in $\mathbb{R}^{6}$. The set of real solutions, say $S$, is readily computed using homotopy continuation.

The Hilbert function is $1,6,12,12, \ldots$ with $I(S)$ being generated by $I(S)_{\leq 2}$. In particular, $I(S)_{\leq 2}$ is a linear space spanned by the linear $f_{1}$ and 15 quadratic ${ }^{2}$

Trivially, $f_{1} \in \sqrt[\mathbb{R}]{\left\langle f_{1}, \ldots, f_{6}\right\rangle}$ and the quadratics are shown to be in the real radical using $\alpha \leq 2$. This computation validates that $\mathcal{V}_{\mathbb{R}}(f)$ consists of 12 points. Moreover, this data matches that displayed in [41, Table 4].

\subsection{High degree}

Our next example was also presented in 41 which was modeled after a system originally from 36 , Sec 4.3]:

$$
f=\left\{\begin{array}{c}
5 x_{1}^{9}-6 x_{1}^{5} x_{2}+x_{1} x_{2}^{4}+2 x_{1} x_{3} \\
-2 x_{1}^{6} x_{2}+2 x_{1}^{2} x_{2}^{3}+2 x_{2} x_{3} \\
x_{1}^{2}+x_{2}^{2}-0.265625
\end{array}\right\} .
$$

We note that the original system in 36 had the term $-6 x_{1}^{5} x_{2}^{2}$ while the system from 41 has the term $-6 x_{1}^{5} x_{2}$. The system $f=0$ has 8 real solutions among the 20 complex solutions. Our computations produce a generating set consisting of 2 quadratics and 10 cubic polynomials for the ideal defined the 8 real solutions which are shown to be in the real radical using $\alpha \leq 2$.

\subsection{Seiler system}

As an illustration of our approach on a problem considered in [49, Ex. 5], namely the Seiler system [59]

$$
f=\left\{\begin{array}{c}
x_{3}^{2}+x_{2} x_{3}-x_{1}^{2} \\
x_{1} x_{3}+x_{1} x_{2}-x_{3} \\
x_{2} x_{3}+x_{2}^{2}+x_{1}^{2}-x_{1}
\end{array}\right\} .
$$

\footnotetext{
${ }^{2}$ Available at www.nd.edu/ ${ }^{\sim}$ aliddel1/validate-reals.
}

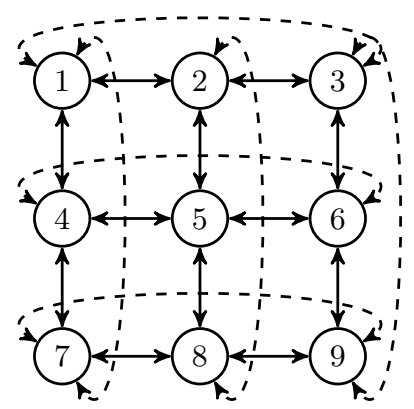

Figure 1: Nearest-neighbor coupling for a $3 \times 3$ grid of nodes.

This system does not have a Pommaret basis with respect to the total degree ordering defined by $x_{1}<x_{2}<x_{3}[59]$. Thus, 49 uses a change of coordinates to overcome this.

Even though $f$ consists of 3 polynomials in 3 variables, $\mathcal{V}_{\mathbb{C}}(f)$ is actually a curve. In particular, $I=\langle f\rangle$ is a onedimensional prime ideal, i.e., $I=\sqrt{I}$ and $\mathcal{V}_{\mathbb{C}}(I)$ is an irreducible curve. Hence, we know that $I=\sqrt[\mathbb{R}]{I}$ if we can compute a real point $x \in \mathcal{V}_{\mathbb{R}}(I)$ which is smooth with respect to $f$, i.e., the rank of $J f(x)$ is 2 .

To that end, we utilize a gradient-descent homotopy 25]. We took $y=(1,-3 / 2,3 / 4)$ and considered the homotopy

$$
H(x, \lambda, t)=\left[\begin{array}{c}
f(x)-t \cdot f(y) \\
\lambda_{0}(x-y)+\lambda_{1} \nabla f_{1}(x)+\lambda_{2} \nabla f_{2}(x)+\lambda_{3} \nabla f_{3}(x)
\end{array}\right]
$$

where $\lambda \in \mathbb{P}^{3}$. Starting at $x=y$ and $\lambda=[1,0,0,0] \in \mathbb{P}^{3}$ when $t=1$, we obtain a point, which is approximately $(0.7009,-0.2504,-0.5868)$, that lies on $\mathcal{V}_{\mathbb{R}}(f)$ and is indeed a smooth point on $\mathcal{V}_{\mathbb{C}}(f)$. Hence, the isosingular set of this point with respect to $f$ is $\mathcal{V}_{\mathbb{C}}(f)$ showing that $I=\sqrt[\mathbb{R}]{I}$.

\subsection{An energy landscape}

Our last example aims to compute the real critical points of the energy landscape of the two-dimensional nearest-neighbor $\phi^{4}$ model on a $3 \times 3$ grid as in 21, 53. We label the nodes $1, \ldots, 9$ with Figure 1 showing the coupling between the nodes. Let $N(i)$ denote the four nearest neighbors of node $i$, e.g., $N(1)=\{2,3,4,7\}$. After selecting various parameters for this model, we consider the potential energy

$$
V(x)=\sum_{i=1}^{9}\left[\frac{1}{40} x_{i}^{4}-x_{i}^{2}+\frac{1}{4} \sum_{j \in N(i)}\left(x_{i}-x_{j}\right)^{2}\right] .
$$

The system defining the critical points is $f=\nabla V$ so that

$$
f_{i}=\frac{1}{10} x_{i}^{3}-2 x_{i}+\sum_{j \in N(i)}\left(x_{i}-x_{j}\right)
$$

The system $f$ is a Gröbner basis and the set $\mathcal{V}_{\mathbb{C}}(f)$ consists of $3^{9}=19,683$ points. However, when searching for real stationary points, one only obtains 3 points, namely

$$
S=\{(0,0,0,0,0,0,0,0,0), \pm(w, w, w, w, w, w, w, w, w)\}
$$

where $w=\sqrt{20} \approx 4.4721$. Hence, $I(S)$ is generated by

$$
G=\left\{x_{1}\left(x_{1}^{2}-20\right), x_{2}-x_{1}, \ldots, x_{9}-x_{1}\right\} .
$$

All nine basis elements were found to be in $\sqrt[\mathbb{R}]{\langle f\rangle}$ with $\alpha=$ $1,2,2, \ldots, 2$, respectively. Therefore, $S=\mathcal{V}_{\mathbb{R}}(f)$, i.e., the energy landscape $V$ has exactly three real critical points. 


\section{KNOWN LIMITATIONS}

As a theoretical approach to computing real radicals using sums of squares, the major practical limitation is based on the value of $\alpha$ needed in (3). To prevent prevent arbitrarily long runtimes, Procedure 1 uses an upper bound $\alpha_{\max }$ which we hope will be replaced in the future by an a priori upper bound based on the input system $f$.

When using floating-point computations as in the examples above, one needs to be cognizant of the effects of roundoff error and conditioning in the computations. We can aim to control this using adaptive precision computations with numerical approximations of solutions that can be refined to arbitrary accuracy.

Systems phrased with numerical approximations of exact numbers can present problems in practice. Fundamentally, these systems violate the model of computation as exact numbers should be input exactly. Hence, representing $1 / 3$ as 0.3333 means that the user is solving a different system from using $1 / 3$. Nonetheless, the robustness and aspects of conditioning of this approach will be explored in future work.

\section{CONCLUSION}

By combining numerical algebraic geometry with sums of squares programming, we have produced a method for certifying that a set of polynomials generate the real radical. The set of polynomials arises from the generators of a set $S$ which is contained in the Zariski closure of the set of real solutions. As considered in 18, combining numerical algebraic geometry and semidefinite programming can potentially improve the efficiency of computations and produce new approaches, in particular for computing and analyzing the set of real solutions of a system of polynomial equations.

\section{REFERENCES}

[1] P. Aubry, F. Rouillier, and M. Safey El Din. Real solving for positive dimensional systems. J. Symbolic Comput., 34(6):543-560, 2002.

[2] B. Bank, M. Giusti, J. Heintz, and G.M. Mbakop. Polar varieties and efficient real elimination. Mathematische Zeitschrift, 238(1):115-144, 2001.

[3] D.J. Bates, D.A. Brake, J.D. Hauenstein, A.J. Sommese, and C.W. Wampler. On computing a cell decomposition of a real surface containing infinitely many singularities. In Mathematical Software - ICMS 2014, volume 8592 of Lecture Notes in Computer Science, pages 246-252. Springer, 2014

[4] D.J. Bates, W. Decker, J.D. Hauenstein, C. Peterson, G. Pfister, F.-O. Schreyer, A.J. Sommese, and C.W. Wampler. Comparison of probabilistic algorithms for analyzing the components of an affine algebraic variety. Appl. Math. Comput., 231:619-633, 2014.

[5] D.J. Bates, J.D. Hauenstein, T.M. McCoy, C. Peterson, and A.J. Sommese. Recovering exact results from inexact numerical data in algebraic geometry. Experimental Mathematics, 22(1):38-50, 2013.

[6] D.J. Bates, J.D. Hauenstein, C. Peterson, and A.J. Sommese. A numerical local dimension test for points on the solution set of a system of polynomial equations. SIAM Journal on Numerical Analysis, 47(5):3608-3623, 2009.

[7] D.J. Bates, J.D. Hauenstein, A.J. Sommese, and C.W. Wampler. Numerically solving polynomial systems with Bertini, volume 25. SIAM, 2013.

[8] E. Becker and R. Neuhaus. Computation of real radicals of polynomial ideals. In Computational algebraic geometry, pages 1-20. Springer, 1993.
[9] E. Becker and T. Wörmann. Radical computations of zero-dimensional ideals and real root counting. Mathematics and Computers in Simulation, 42(4):561-569, 1996.

[10] C. Beltrán and A. Leykin. Robust certified numerical homotopy tracking. Found. Comput. Math., 13(2):253-295, 2013.

[11] G.M. Besana, S. DiRocco, J.D. Hauenstein, A.J. Sommese, and C.W. Wampler. Cell decomposition of almost smooth real algebraic surfaces. Numerical Algorithms, 63(4):645-678, 2013.

[12] L. Blum, M. Shub, and S. Smale. On a theory of computation and complexity over the real numbers: $n p$-completeness, recursive functions and universal machines. Bulletin (New Series) of the American Mathematical Society, 21(1):1-46, 1989.

[13] J. Bochnak, M. Coste, and M.-F. Roy. Real algebraic geometry, volume 36 of Ergebnisse der Mathematik und ihrer Grenzgebiete (3) [Results in Mathematics and Related Areas (3)]. Springer-Verlag, Berlin, 1998. Translated from the 1987 French original, Revised by the authors.

[14] D.A. Brake, D.J. Bates, W. Hao, J.D. Hauenstein, A.J. Sommese, and C.W. Wampler. Bertini_real: Software for one-and two-dimensional real algebraic sets. In Mathematical Software-ICMS 2014, pages 175-182. Springer, 2014.

[15] D.A. Brake, J.D. Hauenstein, and A.J. Sommese. Numerical local irreducible decomposition. To appear in $L N C S$.

[16] M.-D. Choi, T.Y. Lam, and B. Reznick. Sums of squares of real polynomials. In Proceedings of Symposia in Pure mathematics, volume 58, pages 103-126. American Mathematical Society, 1995.

[17] E.K.P. Chong and S.H. Zak. An introduction to optimization, volume 76. John Wiley \& Sons, 2013.

[18] D. Cifuentes and P.A. Parrilo. Sampling algebraic varieties for sum of squares programs. arXiv:1511.06751, 2015.

[19] B.H. Dayton and Z. Zeng. Computing the multiplicity structure in solving polynomial systems. In ISSAC'05, pages 116-123 (electronic). ACM, New York, 2005.

[20] J.P.K. Doye and D.J. Wales. Saddle points and dynamics of lennard-jones clusters, solids, and supercooled liquids. The Journal of Chemical Physics, 116(9):3777-3788, 2002.

[21] R. Franzosi, L. Casetti, L. Spinelli, and M. Pettini. Topological aspects of geometrical signatures of phase transitions. Phys. Rev. E, 60:R5009-R5012, Nov 1999.

[22] P. Gianni, B. Trager, and G. Zacharias. Gröbner bases and primary decomposition of polynomial ideals. Journal of Symbolic Computation, 6(2):149-167, 1988.

[23] A. Griewank and M. R. Osborne. Newton's method for singular problems when the dimension of the null space is $>$ 1. SIAM J. Numer. Anal., 18(1):145-149, 1981.

[24] A. Griewank and M. R. Osborne. Analysis of Newton's method at irregular singularities. SIAM J. Numer. Anal., 20(4):747-773, 1983.

[25] Z.A. Griffin and J.D. Hauenstein. Real solutions to systems of polynomial equations and parameter continuation. Adv. Geom., 15(2):173-187, 2015.

[26] Z.A. Griffin, J.D. Hauenstein, C. Peterson, and A.J. Sommese. Numerical computation of the Hilbert function and regularity of a zero dimensional scheme. In Connections between algebra, combinatorics, and geometry, volume 76 of Springer Proc. Math. Stat., pages 235-250. Springer, New York, 2014.

[27] J.D. Hauenstein. Numerically computing real points on algebraic sets. Acta applicandae mathematicae, 125(1):105-119, 2013.

[28] J.D. Hauenstein and Sommese A.J. What is numerical algebraic geometry? J. Symb. Comput., To appear. 
[29] J.D. Hauenstein, I. Haywood, and A.C. Liddell, Jr. An a posteriori certification algorithm for newton homotopies. In Proceedings of the 39th International Symposium on Symbolic and Algebraic Computation, ISSAC '14, pages 248-255, New York, NY, USA, 2014. ACM.

[30] J.D. Hauenstein and A.C. Liddell Jr. Certified predictor-corrector tracking for newton homotopies. Journal of Symbolic Computation, 74:239 - 254, 2016.

[31] J.D. Hauenstein, B. Mourrain, and A. Szanto. Certifying isolated singular points and their multiplicity structure. In ISSAC'15-Proceedings of the 2015 ACM International Symposium on Symbolic and Algebraic Computation, pages 213-220. ACM, New York, 2015.

[32] J.D. Hauenstein, A.J. Sommese, and C.W. Wampler. Regenerative cascade homotopies for solving polynomial systems. Appl. Math. Comput., 218(4):1240-1246, 2011.

[33] J.D. Hauenstein and F. Sottile. Algorithm 921: alphaCertified: certifying solutions to polynomial systems. ACM Transactions on Mathematical Software (TOMS), $38(4): 28,2012$

[34] J.D. Hauenstein and C.W. Wampler. Isosingular sets and deflation. Found. Comput. Math., 13(3):371-403, 2013.

[35] I. Janovitz-Freireich, B. Mourrain, L. Rónyai, and Á. Szántó. On the computation of matrices of traces and radicals of ideals. Journal of Symbolic Computation, 47(1):102-122, 2012.

[36] R.B. Kearfott. Some tests of generalized bisection. ACM Transactions on Mathematical Software (TOMS), 13(3):197-220, 1987.

[37] C.T. Kelley. Iterative methods for optimization, volume 18 of Frontiers in Applied Mathematics. Society for Industrial and Applied Mathematics (SIAM), Philadelphia, PA, 1999.

[38] C.T. Kelley. Solving nonlinear equations with Newton's method. SIAM, Philadelphia, 2003.

[39] T. Krick and A. Logar. An algorithm for the computation of the radical of an ideal in the ring of polynomials. In Applied algebra, algebraic algorithms and error-correcting codes, pages 195-205. Springer, 1991.

[40] J.B. Lasserre, M. Laurent, B. Mourrain, P. Rostalski, and P. Trébuchet. Moment matrices, border bases and real radical computation. Journal of Symbolic Computation, 51:63-85, 2013.

[41] J.B. Lasserre, M. Laurent, and P. Rostalski. Semidefinite characterization and computation of zero-dimensional real radical ideals. Foundations of Computational Mathematics, 8(5):607-647, 2008.

[42] J.B. Lasserre, M. Laurent, and P. Rostalski. A prolongation-projection algorithm for computing the finite real variety of an ideal. Theoretical Computer Science, 410(27):2685-2700, 2009.

[43] J.B. Lasserre, M. Laurent, and P. Rostalski. A unified approach to computing real and complex zeros of zero-dimensional ideals. In Emerging applications of algebraic geometry, pages 125-155. Springer, 2009.

[44] M. Laurent and P. Rostalski. The approach of moments for polynomial equations. In Handbook on Semidefinite, Conic and Polynomial Optimization, pages 25-60. Springer, 2012.

[45] B. Lesieutre and D. Wu. An efficient method to locate all the load flow solutions - revisited. In 53rd Annual Allerton Conf. Commun., Control, and Comput., Sept. 29 - Oct. 2 2015.

[46] A. Leykin, J. Verschelde, and A. Zhao. Newton's method with deflation for isolated singularities of polynomial systems. Theoret. Comput. Sci., 359(1-3):111-122, 2006.

[47] Y. Lu, D.J. Bates, A.J. Sommese, and C.W. Wampler. Finding all real points of a complex curve. In Algebra, Geometry and their Interactions, volume 448 of Contemporary Mathematics, pages 183-205, 2007.

[48] W. Ma and J.S. Thorp. An efficient algorithm to locate all the load flow solutions. IEEE Transactions on Power Systems, 8(3):1077-1083, Aug 1993.
[49] Y. Ma, C. Wang, and L. Zhi. A certificate for semidefinite relaxations in computing positive-dimensional real radical ideals. Journal of Symbolic Computation, 2014.

[50] M. Marshall. Positive polynomials and sums of squares, volume 146 of Mathematical Surveys and Monographs. American Mathematical Society, Providence, RI, 2008.

[51] D. Mehta, T. Chen, J.D. Hauenstein, and D.J. Wales. Communication: Newton homotopies for sampling stationary points of potential energy landscapes. The Journal of Chemical Physics, 141(12):121104, 2014.

[52] D. Mehta, T. Chen, J.W.R Morgan, and D.J. Wales. Exploring the potential energy landscape of the Thomson problem via Newton homotopies. The Journal of Chemical Physics, 142(19):194113, 2015.

[53] D. Mehta, J.D. Hauenstein, and M. Kastner. Energy-landscape analysis of the two-dimensional nearest-neighbor $\varphi^{4}$ model. Phys. Rev. E, 85:061103, Jun 2012.

[54] R. Neuhaus. Computation of real radicals of polynomial ideals - ii. Journal of Pure and Applied Algebra, 124(1):261-280, 1998

[55] A. Papachristodoulou, J. Anderson, G. Valmorbida, S. Prajna, P. Seiler, and P.A. Parrilo. SOSTOOLS: Sum of squares optimization toolbox for MATLAB. http://arxiv.org/abs/1310.4716, 2013. Available from http://www.eng.ox.ac.uk/control/sostools, http://www.cds.caltech.edu/sostools and http://www.mit.edu/ parrilo/sostools.

[56] F. Rouillier, M.-F. Roy, and M. Safey El Din. Finding at least one point in each connected component of a real algebraic set defined by a single equation. J. Complexity, 16(4):716-750, 2000.

[57] M. Safey El Din and É. Schost. Polar varieties and computation of one point in each connected component of a smooth real algebraic set. In Proceedings of the 2003 international symposium on Symbolic and algebraic computation, pages 224-231. ACM, 2003.

[58] A. Seidenberg. A new decision method for elementary algebra. Ann. of Math. (2), 60:365-374, 1954.

[59] W. Seiler. Involution - the formal theory of differential equations and its applications in computer algebra and numerical analysis. Habilitation thesis. University of Mannheim, 2002.

[60] S. Smale. Newton's method estimates from data at one point. Springer, 1986.

[61] A.J. Sommese and J. Verschelde. Numerical homotopies to compute generic points on positive dimensional algebraic sets. J. Complexity, 16(3):572-602, 2000. Complexity theory, real machines, and homotopy (Oxford, 1999).

[62] A.J. Sommese and C.W. Wampler. Numerical algebraic geometry. In The mathematics of numerical analysis (Park City, UT, 1995), volume 32 of Lectures in Appl. Math., pages 749-763. Amer. Math. Soc., Providence, RI, 1996.

[63] A.J. Sommese and C.W. Wampler, II. The numerical solution of systems of polynomials arising in engineering and science. World Scientific Publishing Co. Pte. Ltd., Hackensack, NJ, 2005.

[64] S.J. Spang. On the computation of the real radical. PhD thesis, Thesis, Technische Universität Kaiserslautern, 2007.

[65] G. Stengle. A nullstellensatz and a positivstellensatz in semialgebraic geometry. Math. Ann., 207:87-97, 1974.

[66] B. Xia and L. Yang. An algorithm for isolating the real solutions of semi-algebraic systems. Journal of Symbolic Computation, 34(5):461-477, 2002.

[67] G. Zeng. Computation of generalized real radicals of polynomial ideals. Science in China Series A: Mathematics, 42(3):272-280, 1999. 